\title{
CMOS-MEMS 8-BIT MEMDAC NANOPOSITIONER WITH INTEGRATED POSITION SENSING AND DIGITAL CONTROL \\ Neil Sarkar ${ }^{1 *}$ and Raafat Mansour ${ }^{1}$ \\ ${ }^{1}$ University of Waterloo, Waterloo, Ontario, Canada
}

\begin{abstract}
An 8-bit MEMDAC (microelectromechanical digital-toanalog converter) with nanometric position sensing and digital control has been implemented in a CMOS-MEMS process. The device is intended for use in scanning probe lithography and requires a closed-loop position resolution of $1 \mathrm{~nm}$. Lateral electrothermal actuators provide a displacement of $8 \mu \mathrm{m}$ to each of the 8 inputs of the compliance network. The step size corresponding to a transition of the least significant bit (LSB) is $\sim 31 \mathrm{~nm}$. A piezoresistive sensor integrated in the LSB has an output-referred position resolution of $0.39 \mathrm{~nm}$. The design has been extended to 2 dimensions with an integrated cantilever and CMOS electronics to reduce pincount.
\end{abstract}

\section{INTRODUCTION}

Arrays of MEMS-based nanopositioners are desirable for parallel atomic precision patterning (APP) on Si. [1] The $\mathrm{H}$ depassivation lithography process is depicted below in figure 1 . The lateral position resolution, stiffness, range of motion, voltage limitations, stability, and vacuum compatibility requirements of the APP process are used as specifications in the design of the MEMDAC positioning unit presented here.

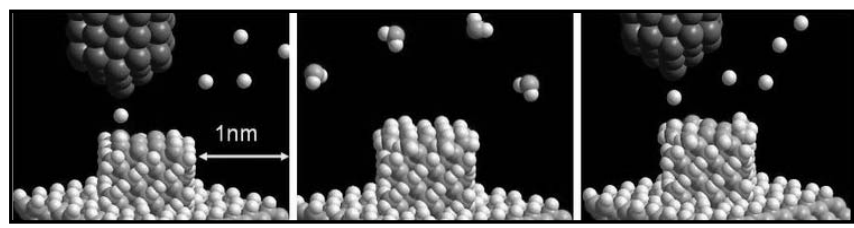

Figure 1: H Depassivation lithography process begins with the removal of $H$ from a Si surface with atomic precision (left). In the deposition phase (center), $\mathrm{SiH}_{2}$ radicals in the gas phase deposit one Si atom wherever an $\mathrm{H}$ atom is removed (patterned Atomic Layer Epitaxy). After the deposition cycle, $\mathrm{SiH}_{2}$ is evacuated and the pattering step is repeated to create $3 D$ structures.

We report an 8-bit MEMDAC that is fabricated in the ASIMPS (Application-specific integrated MEMS processes) CMOS-MEMS process [2]. The design is based on previously reported MEMDAC architectures [3,4,5] and has been augmented in the following ways:

1) The number of bits has been increased to 8 in order to achieve a step-size of $31 \mathrm{~nm}$.

2) The LSB contains a piezoresistive position sensor for sub-positioning within a bit. The piezoresistive position sensor has an output-referred resolution of $<1 \mathrm{~nm}$.

3) A CMOS scan chain and current drivers are integrated with the device in order to control multiple MEMDACs with a 5-wire interface.

4) A latch has been placed at the distal end of the positioner for power-off positioning.

5) A 2-D positioner with an integrated cantilever has been implemented using four 1-D MEMDACs

\section{DEVICE DESIGN}

\section{Lateral Actuation}

Electrothermal actuation based on the thermal bimorph effect can be implemented in a CMOS-MEMS process by laterally offsetting the internal layers in a structural beam, as shown in figure 2. The details of the actuator's design and optimization have been presented elsewhere [6].

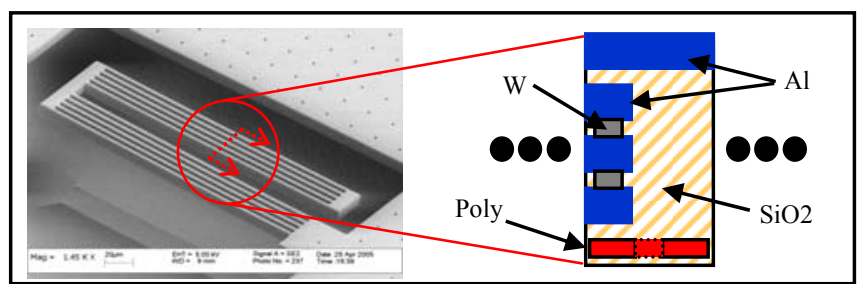

Figure 2: SEM image (left) of a lateral actuator in the ASIMPs CMOS-MEMS process. The parallel arrangement of beams increases the output force. A cross section of one bimorph (right) shows the offset in the internal metal layers used to achieve a lateral thermal moment.

The two constituent materials of the bimorph are aluminum and silicon dioxide, which have a similar young's modulus but a very dissimilar TCE $\left(\alpha_{\mathrm{Al}}=23.1 \mu \mathrm{K}^{-1}\right.$ and $\left.\alpha_{\mathrm{SiO} 2}=0.5 \mu \mathrm{K}^{-1}\right)$.

It is advantageous to couple multiple bimorphs together in the actuator in order to increase out-of-plane stiffness and lateral force; however, the bimorph beams cannot simply be coupled to a rigid plate, since this would impose a zero-moment constraint and result in zero-deflection of the beams. Arranging 2 bimorphs (in series within a beam) in a symmetrically opposed fashion eliminates rotation at the distal end of the beam, thus enabling the designer to couple multiple beams together to a plate. Due to the asymmetric cross section of the beam in the out-of-plane (z) direction, a single set of coupled beams experiences parasitic out-of-plane motion. This issue is mitigated by folding and reflecting the first set of beams, resulting in the U-shaped structure of figure $3 \mathrm{c}$.

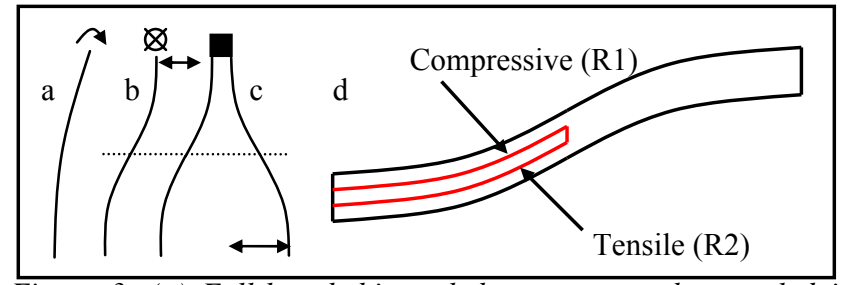

Figure 3: (a) Full-length bimorph beams cannot be coupled in parallel due to the moment that exists at the tip. (b) A pair of symmetrically opposed bimorphs in a beam has parasitic out-ofplane motion. (c) This u-shaped arrangement moves in a purely rectilinear fashion. (d) Piezoresistors are differentially arranged within a beam to sense lateral deflection. 
This type of lateral actuator was selected due to its relatively high work/unit volume metric, its capability to produce a large static displacement, and its low driving voltage which is CMOS compatible. In vacuum, this actuator is capable of deflecting $10 \mu \mathrm{m}$ while consuming $1.3 \mathrm{~mW}$.

\section{Lateral Piezoresistive Position Sensing}

The gauge factor of the polysilicon available in the ASIMPs process has been extracted using a passive test structure in conjunction with a motion control stage. An FEA model of the passive cantilever used in the experiment is used to predict the strain in the polysilicon layer as a function of deflection. The measured resistance of the piezoresistors is then applied to this FEA model in order to identify the gauge factor. Our measurements indicate a gauge factor of $\sim 20$ in the high-valueresistor-type polysilicon available in the process. This number is significantly higher than the gauge factors obtained in the silicided and low-value-resistor materials available in the same process.

In order to obtain strain measurements on lateral displacements while mitigating TCR (temperature coefficient of resistivity) effects, we adopt the design pattern shown in figure 3. Within a monolithic beam structure, we place 2 piezoresistors in parallel, on either side of the beam's neutral axis. Upon actuation, one of the resistors experiences compressive stress while the other experiences tensile stress, while they both experience the same temperature distribution. The cross-sectional temperature profile at any plane along the length of the beam is assumed to be equithermal. Thus, this design is insensitive to temperature variations along the length of the beam, since the temperature distribution within the 2 resistors is identical. Measurements on piezoresistors incorporated into an actuator beam are reported elsewhere [7]. Measurements of piezoresistance in the ASIMPs process are also presented in [8]. In this work, we integrate the piezoresistors within the MEMDAC's compliance network, as discussed further below.

\section{Compliance Network}

The MEMDAC design consists of a network of compliant beams that is analogous to the resistive ladder network that is present in an R-2R electronic DAC, as described in [3] and shown in figure 4.

The actuators that provide input displacements into the compliance network are limited by hard-stops in order to provide distinct positions of $0 \mu \mathrm{m}$ and $8 \mu \mathrm{m}$. A lateral self-assembly displacement occurs in the actuators due to residual stress gradients, so the hard-stops are judiciously shifted to mitigate this effect. In placing the hard-stops, one must also consider thermal coupling from adjacent actuators through the compliance network, substrate, and ground shield. These sources of heat flow result in partial actuation of a bit that is intended to be "off" when adjacent bits are "on." The hard stops must therefore be placed conservatively so that this partial actuation does not displace the actuator from its "off" position.

An additional actuator is placed orthogonally with respect to the output of the MEMDAC, and serves as a latching element. This actuator self-assembles into the engaged position upon release, eliminating power consumption while the device is off.

The LSB in the MEMDAC connects to a load beam with integrated differential piezoresistive strain sensors. This geometry ensures that the resistors are temperature-matched, but they experience opposite stress. An off-chip bridge circuit is used to

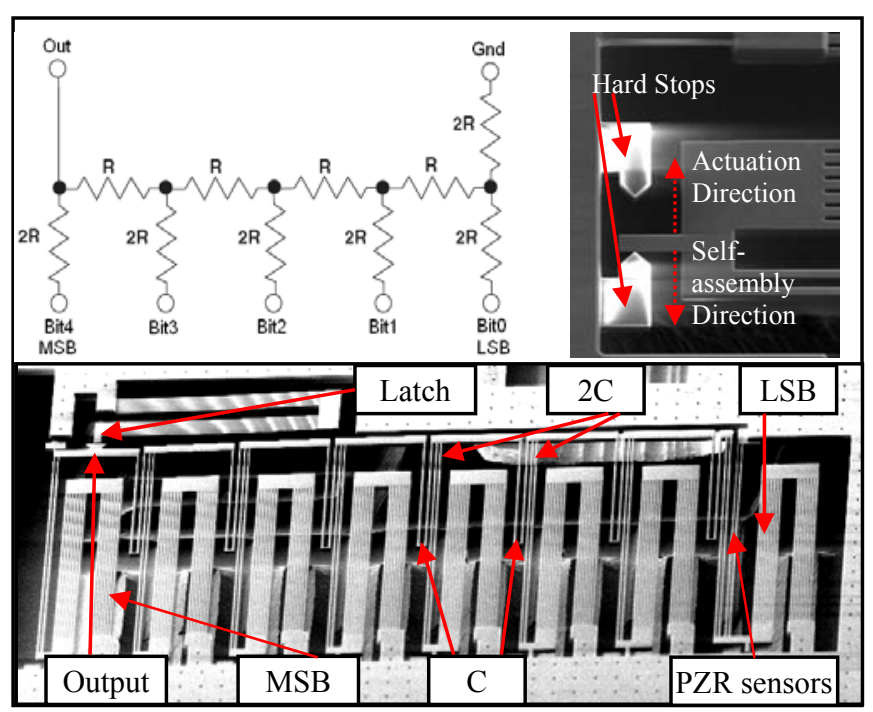

Figure 4: Electronic R-2R DAC (top left) and MEMS implementation (bottom). SEM image shows beams of compliance $C$ and $2 C$ in the network. A latch is present for power-off positioning at the output, and the LSB and MSB are indicated. The piezoresistive strain gauge is located within the load beam near the LSB. Hard stops are used to limit the input position to 2 distinct values (top right).

measure position with a resolution of $100 \mathrm{~nm}$. A $100 \mathrm{~nm}$ position resolution in the LSB corresponds to $<0.5 \mathrm{~nm}$ displacement at the output.

\section{ANALYSIS}

\section{Electrical Equivalent Circuit}

An equivalent circuit of the mechanical system is developed according to the methodology presented in [9] and shown in figure 5. In the simplest case, the beams are considered to be pure springs and are represented as capacitors with a value of:

$$
C_{e}=\frac{1}{k_{m}}=C_{m}
$$

Where

$C_{e}=$ capacitance in the electrical domain

$k_{m}=$ stiffness in the mechanical domain

$C_{m}=$ compliance in the mechanical domain

In this analogy (the $\mathrm{e} \rightarrow \mathrm{V}$ convention), the input and output displacements of beams are replaced by input and output charges on capacitors; the output voltage is calculated after charge sharing has occurred, and represents the output force. The output force is applied to the load beam, resulting in an output displacement that is equivalent to the charge that is obtained with:

$$
Q_{\text {net }}=C * V_{\text {out }}
$$

Where

$Q_{n e t}$ is the net charge across the network

$C$ is the capacitance looking into the network (this value remains constant regardless of the number of bits in the network)

$V_{\text {out }}$ is the voltage at the output of the circuit, and is analogous to the force at the output of the mechanical system. 


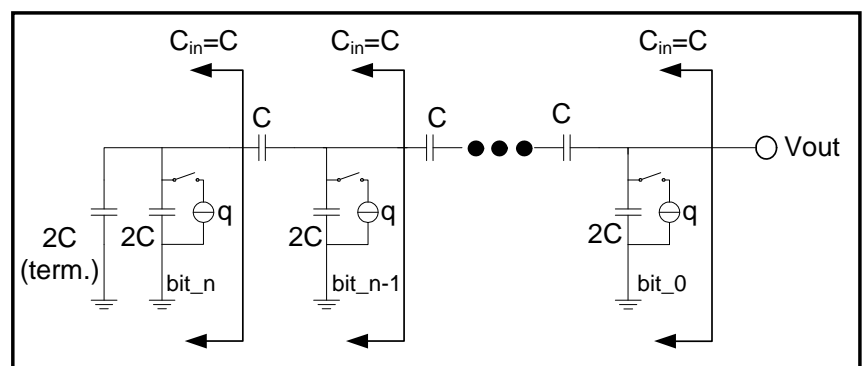

Figure 5: Mechanical equivalent circuit of the compliance network. Input displacements are represented by charge sources q. Spring constants of beams are replaced with capacitances $C$ and 2C. The capacitance looking into the network is always $C$, regardless of the number of bits in the system.

Note that this convention captures the fact that the operation of the MEMDAC is non-dissipative in the mechanical domain, since it uses capacitances to represent stiffness, as opposed to resistances that would represent dampers.

\section{Geometric Advantage}

The MEMDAC's compliance network provides a geometric advantage of $1 / 256$ between the LSB and the output, as calculated by applying a charge of $\mathrm{q}$ to the LSB and obtaining an output charge of $\mathrm{q} / 256$. This is an attractive feature and is indeed the reason why such an architecture was considered for the atomically precise patterning application. Several sources of noise limit our position resolution in the piezoresistive sensor to $\sim 100 \mathrm{~nm}$. This resolution is effectively scaled down by a factor of 256 in the compliance network. Thus, we obtain superior output-referred resolution by including the sensor in the LSB of the device.

The total achievable output displacement is sensitive to the number of bits, and can be obtained with the following relation that is easily calculated from the R-2R ladder circuit:

$$
D_{\max }=D_{a c t} * \sum_{i=1}^{N} \frac{1}{2^{i}}
$$

Where

$D_{\max }$ is the maximum output displacement

$D_{a c t}$ is the deflection of each bit $(8 \mu \mathrm{m})$

$N$ is the number of bits

\section{Mechanical Advantage}

The output stiffness of the MEMDAC, represented by the capacitance looking into the network, remains constant regardless of the number of bits as illustrated in figure 5. Each bit in the compliance network experiences a binary weighted mechanical advantage equivalent to the reciprocal of its geometric advantage.

The mechanical and geometric advantage features can also be implemented by connecting a single actuator to its load through a pair of springs (connected similarly to a single unit in the $\mathrm{C}-2 \mathrm{C}$ ladder), but with a stiffness ratio of $1 / 256$. This would result in a more compact design than the MEMDAC. Such a design pattern would result in improved output-referred sensor resolution and enhanced stiffness at the expense of reduced deflection.

\section{Thermomechanical Noise}

The thermomechanical noise that is introduced by the network between the LSB and the output is lower than the desired resolution. In order to calculate this, a damping term must be included in the beam model. The full model of the beam as a mass-spring-damper system is replaced as in [9] with an equivalent
RLC circuit. Thermomechanical noise is the mechanical equivalent of electrical Johnson noise, and can be calculated with the following expression from [10]:

$$
\left|Z_{n}(f)\right|=\sqrt{4 k_{b} T B} / k
$$

Where

$Z_{n}(f)$ is the noise displacement

$k_{b}$ is boltzmann's constant

$T$ is temperature

$B$ is damping

$k$ is the stiffness of the beam

The methodology for calculating Johnson noise in the resistive ladder of an electronic R-2R DAC can be used to calculate the output-referred thermomechanical noise in the compliance network. The damping terms in the compliance network are not binary weighted, in fact they are similar for both the $\mathrm{C}_{\mathrm{m}}$ and $2 \mathrm{C}_{\mathrm{m}}$ beams. The stiffness term does affect the calculation, making it similar to the R-2R case. The schematic below contains only the damping symbol but the expression for noise is scaled according to the beam stiffness. We replace the resistors with a series combination of a resistor and a voltage noise source, and calculate the equivalent voltage noise for the 8-bit $\mathrm{DAC}$ as shown in figure 6. Assuming a bandwidth limited to 10 $\mathrm{kHz}$ by the tunneling amplifier in our system, a typical value for $\mathrm{B}$ of $1 \mathrm{E}-5$, and room temperature operation, this calculation yields a value of $\sim 0.13 \mathrm{~nm}$, which is below the output-referred position resolution of the piezoresistive sensor placed in the LSB.

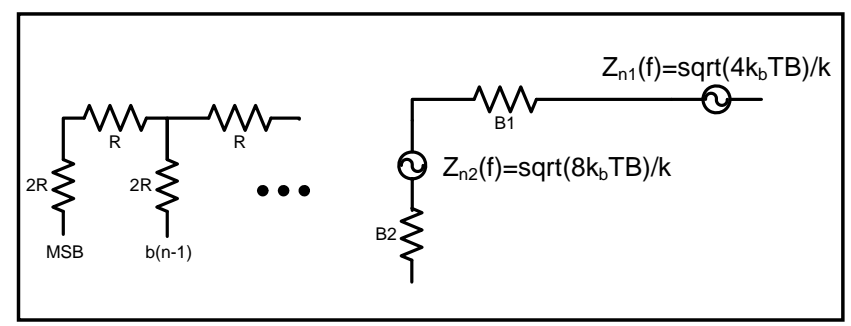

Figure 6: Calculation of Johnson noise in a resistive ladder is similar to calculation of thermomechanical noise in a compliance network where the beams have damping terms $B$.

\section{FEM and LEM}

A finite element model of the compliance network was developed using Coventorware in order to predict the integral and differential nonlinearity error (INL and DNL), (figure 7). This model assumed no mismatch in the beam stiffness. A lumped element model in the electrical domain was implemented using Cadence. This model accounts for stiffness mismatches. Results of both models are included with experimental results, and are shown to capture the nonlinearities that were measured.

It is possible to operate the LSB using an analog input to scan within a step while all other bits are fixed to a specific binary input value. This operation has been modeled, and our FEM and predicts excellent linearity. The position resolution required to characterize this behaviour is beyond the capability of our motion capture system; however, tunneling measurements may confirm this in the future. 


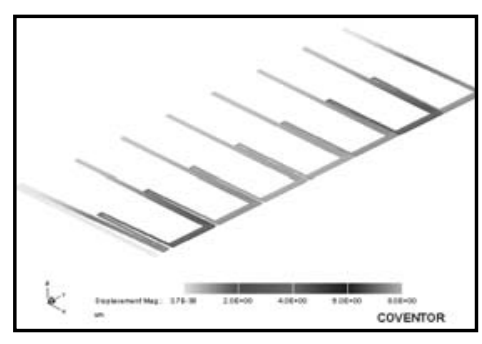

Figure 7: Coventor simulation of the compliance network in a state corresponding to input [01111110]. The MSB is the right-most bit connected to the output shuttle, and the LSB is the left-most bit.

\section{RESULTS \\ 1-D MEMDAC}

Data was taken using a stroboscopic motion capture system (Umech) using an off-chip 8-bit binary counter to drive the actuators in a bitwise incrementing pattern. The results of this test are compared to the LEM and FEM outputs in figure 8.

The maximum measured DNL is $2.3 \mathrm{LSB}$ and the INL was measured at $1.9 \mathrm{LSB}$.

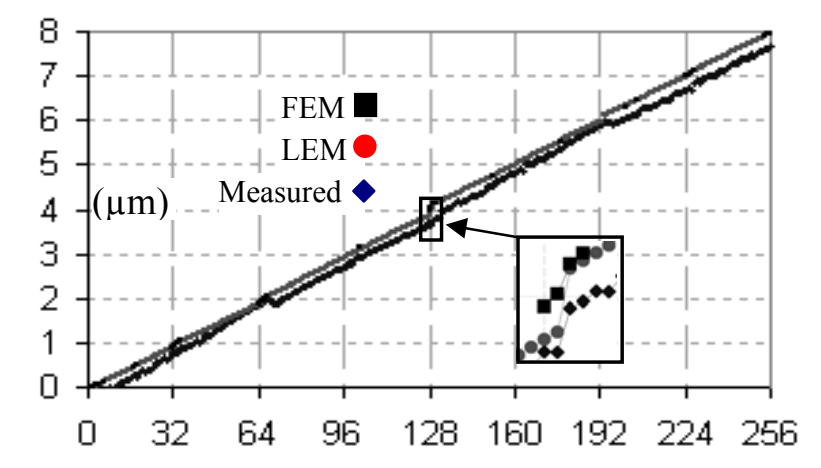

Figure 8: Finite Element Model, Lumped Element Model and measurement results. Note the significant DNL error that occurs at the transition from 127 to 128.

\section{Sources of Error}

Several processing and design aspects of the device contribute to the observed positioning error. Structural bloat that occurs during the post-CMOS release process reduces the range of motion of the actuators. The asymmetry in the $\mathrm{C}$ and $2 \mathrm{C}$ beams contributes to a compliance mismatch that results from a difference in loading effects during etching. Thermal coupling through the ground plane, substrate and compliance network results in parasitic actuation. This effect is most noticeable in the transition from input value 127 to 128 , since these are the two adjacent values with the largest difference in power consumption and temperature.

\section{2-D MEMDAC}

An orthogonal arrangement of four 1-D MEMDACs was used to position a centrally mounted cantilever in 2-D (figure 9). The device can be reliably positioned on a 2-D grid with a $32 \mathrm{~nm}$ step size, and exhibits similar INL and DNL. The rationale behind this device is to position a tip on a 2-D grid quickly with only digital inputs, and scan within each cell in an analog fashion (figure 9).

Two versions of the device were designed, one with integrated CMOS electronics to reduce pincount, and one with 32 bond-wires. The CMOS electronics include a scan chain which serially loads data into electronic R-2R DACs. The DACs are used to set an input voltage to a source follower, which in turn drives the bits of the MEMDAC. The CMOS electronics occupy approximately the same area as the 2-D MEMDAC, but can be reduced significantly with the omission of the DAC circuits for all
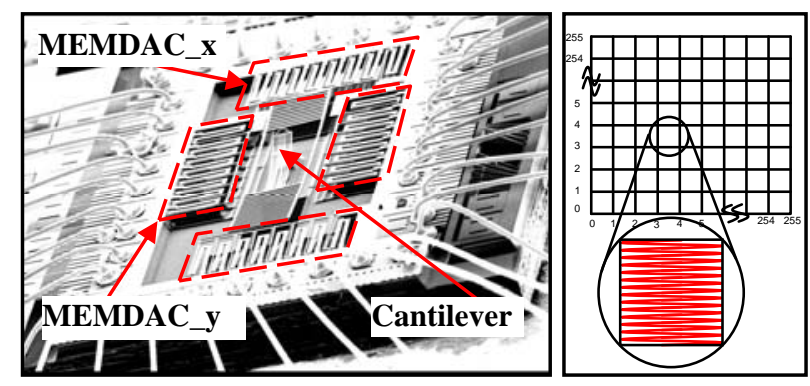

Figure 9: 2-D MEMDAC composed of 4 orthogonally arranged 12-D MEMDAC's. The device addresses a grid and can scan within a cell in closed loop using the LSB positioner.

\section{CONCLUSION}

An 8-bit MEMDAC based on the R-2R electronic DAC architecture was fabricated using a CMOS-MEMS process. The step size achievable with this design is $32 \mathrm{~nm}$, which is smaller than the resolution attainable with a lateral differential piezoresistive position sensing scheme. By placing a strain gauge at the LSB of the device, sub-nanometer position resolution was obtained with measurement levels above the thermal noise floor. Although substep positioning operation exhibits good linearity, significant error is observed during transitions in which a large number of bits are toggling and significantly changing the power consumption and heat generation from the device.

\section{ACKNOWLEDGEMENTS}

This material is based upon work supported by the Defense Advanced Research Project Agency (DARPA) and Space and Naval Warfare Center, San Diego (SPAWARSYSCEN-SD) under contract N66001-08-C-2040, and the Emerging Technology Fund of the State of Texas. The authors would also like to thank Mr. Kyle Trainor for his assistance in the characterization of CMOS electronics.

\section{REFERENCES}

[1] J. N. Randall et. al., "Atomic precision patterning on Si: An opportunity for a digitized process", Microelectronic Engineering, vol. 87, pp. 955-958, Dec. 2009.

[2] Fedder. G. K., et. al., Invited Paper, Proc. IEEE, Vol. 96, No. 2, pp. $306-322$, Feb. 2008.

[3] Toshiyoshi, H. et. al., JMEMS, Vol. 9-2, pp. 218-225, June 2000.

[4] R.Yeh, R.Conant, K.S.J.Pister, "Mechanical Digital-toAnalog Converter," Transducers '99, pp. 998-1001.

[5] N. Sarkar, R. Mansour, "CMOS-MEMS Nanopositioners with Integrated Position Sensors and Digital Control," presented at CWMEMS 2007.

[5] N. Sarkar, R. Mansour, "CMOS-MEMS Nanopositioners with Integrated Position Sensors and Digital Control," presented at CWMEMS 2007.

[6] Gilgunn, P.J. et. al., "CMOS-MEMS Lateral electrothermal actuators," JMEMS, Vol. 17, No. 1, pp. 103-114.

[7] Sarkar, N. et. al., "Modular MEMS experimental platform for TEM," Proc. MEMS 2006.

[8] Liu, J. et. al, "Polysilicon Sensors for CMOS-MEMS Electrothermal Probes," Proc. Transducers 2009, pp.24252428, June 2009.

[9] Senturia, S.D., "Microsystem Design," Kluwer Academic Publishing, 2001.

[10] T. B. Gabrielson, IEEE Electron Devices, 40, 903 (1993).

\section{CONTACT}

*N. Sarkar, nsarkar@uwaterloo.ca 